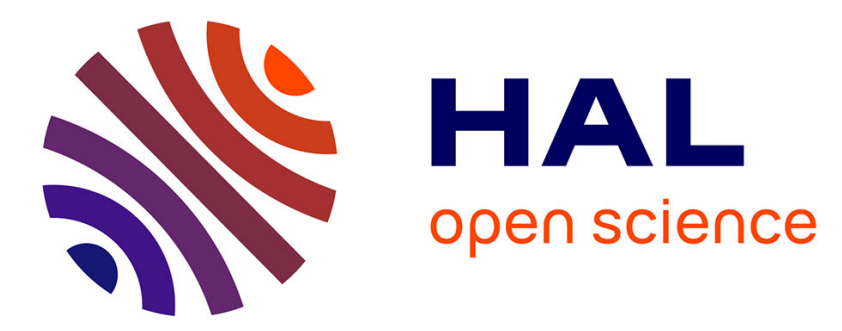

\title{
Seismic imaging of transmission overhead line structure foundations
}

Denis Vautrin, Matthieu Voorons, Jérôme Idier, Yves Goussard, Nicolas Paul

\section{To cite this version:}

Denis Vautrin, Matthieu Voorons, Jérôme Idier, Yves Goussard, Nicolas Paul. Seismic imaging of transmission overhead line structure foundations. Acoustics 2012, Apr 2012, Nantes, France. hal00810880

\section{HAL Id: hal-00810880 \\ https://hal.science/hal-00810880}

Submitted on 23 Apr 2012

HAL is a multi-disciplinary open access archive for the deposit and dissemination of scientific research documents, whether they are published or not. The documents may come from teaching and research institutions in France or abroad, or from public or private research centers.
L'archive ouverte pluridisciplinaire HAL, est destinée au dépôt et à la diffusion de documents scientifiques de niveau recherche, publiés ou non, émanant des établissements d'enseignement et de recherche français ou étrangers, des laboratoires publics ou privés. 


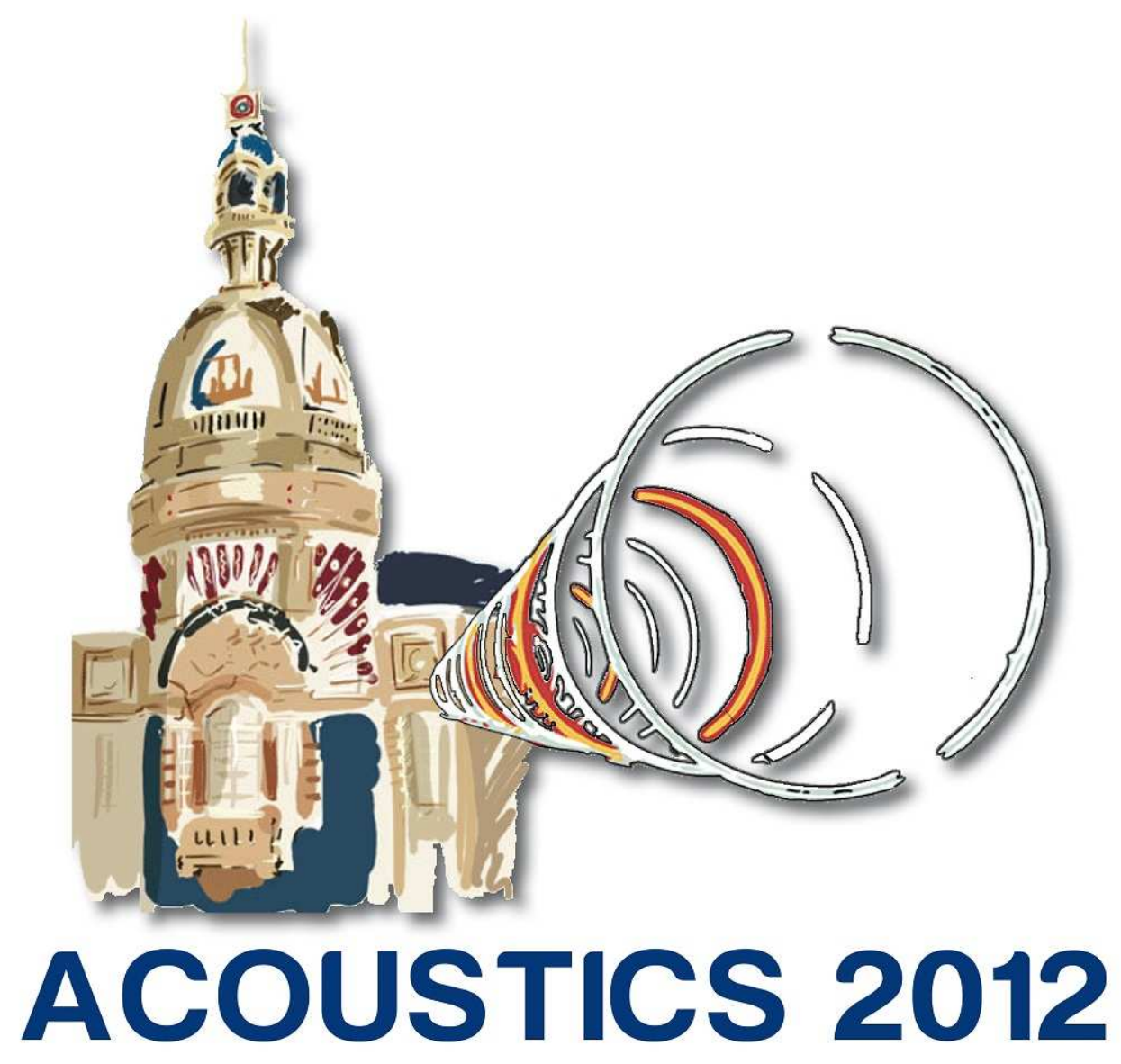

\section{Seismic imaging of transmission overhead line structure foundations}

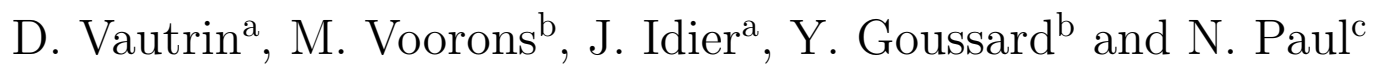

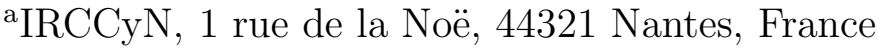

${ }^{\text {b}}$ Ecole Polytechnique de Montreal, Campus de l'Université de Montréal 2500, chemin de Polytechnique Montréal (Québec) H3T 1J4

${ }^{c}$ EDF R\&D, département STEP, 6 quai Watier, 78401 Chatou, France denis.vautrin@irccyn.ec-nantes.fr 
This work deals with the nondestructive testing of foundations by means of a seismic imaging procedure. Our goal is to propose an inversion scheme that determines the shape of the foundations from the measured data with an acceptable computational effort. The inversion procedure was defined from a model based on the linear elastic wave equations in two dimensions. The inverse problem is particularly difficult to solve for several reasons: the distribution of numerous physical characteristics must be estimated, the relation between the underground characteristics and the measured data is highly nonlinear, and this is a large scale and ill conditioned problem. Three inversion techniques are proposed. They minimize a regularized least-square criterion iteratively. The first circumvent the difficulties of the problem by means of algorithmical and mathematical techniques. It favours the reconstruction of smooth areas separated by sharp boundaries. Two additional methods were developed. They take into account more specific characteristics of the problem. Tests were performed on synthetic data. The results show that the first method leads to satisfying results. The addition of more specific priors leads to more precise and faster reconstructions.

\section{Context}

Our work is part of an industrial project initiated by the French Réseau de Transport d'Electricité (RTE). The aim is to determine the geometry of transmission overhead line structure foundations from indirect measurements acquired at the ground surface.

\subsection{Description of the experimental procedure}

The underground medium is probed with a seismic imaging procedure. This nondestructive testing technique consists of (1) the emission of a seismic wave train into the underground medium and (2) the measurement of the resulting wave velocity.

To generate a seismic wave, a vibrator is used [1]. The main advantage of this device is that it enables one to control the temporal signature of the source signal. Its spectral content should be chosen depending on the dimensions of the probed medium and the expected spatial resolution. The emision of high frequency components helps to improve the spatial resolution but low frequency components are also required to retrieve the underground characteristics [2]. In our case, the source signal frequency band ranges from $100 \mathrm{~Hz}$ to $1 \mathrm{kHz}$ approximately.

A set of geophones placed on the ground surface is used to perform the measurements. Geophones record the vertical component of the seismic wave velocity. They are characterized by their corner frequency (it is equal to $100 \mathrm{~Hz}$ in our study). Up to 100 geophones can be used in our study. In the following, the number of geophones is denoted by $N_{g}$.

The whole system is placed on the ground surface near the foudation. The procedure is repeated for multiple locations of the source in order to get richer information. The computation cost would be prohibitive for a three-dimensional imaging. The measurement procedure is thus adapted for a two-dimensional imaging: the whole measurement system is placed along a line, roughly in the plane of symmetry of the foundation.

\subsection{Resolution of the forward problem}

Our goal is to solve the inverse problem. The aim is to determine the foundation geometry knowing the data recorded by the sensors and the experimental procedure (i.e. the signal generated by the source and the location of the vibrator and the geophones).

To solve the inverse problem, an algorithm of resolution of the forward problem is required. The aim is to produce a synthetic set of data knowing the underground characteristics. In our study, this algorithm has been developped by the R\&D division of EDF (Electricité De France). The simulation of the seismic waves propagation through the underground medium is based on a two-dimensional elastic model. In this case, the synthetic data depends on the spatial distribution of three physical characteristics:

- the pressure-wave velocity $v_{p}$;

- the shear-wave velocity $v_{s}$;

- the density $\rho$.

The propagation equations are formulated in the frequential domain and discretized with a finite-difference scheme. In this way, the forward problem is solved at a reasonable computational cost, which reduces the computation requirements for the resolution of the inverse problem. For a given angular frequency $\omega$ and a given source location $k$, a synthetic set of data is denoted by the vector $\boldsymbol{g}_{\omega, k}$ of length $N_{g}$. It is related to the underground characteristics as follows:

$$
\boldsymbol{g}_{\omega, k}\left(\boldsymbol{v}_{p}^{2}, \boldsymbol{v}_{s}^{2}, \boldsymbol{\rho}\right)=\mathbf{E}_{\mathbf{1}} \mathbf{A}_{\omega}^{-1}\left(\boldsymbol{v}_{p}^{2}, \boldsymbol{v}_{s}^{2}, \boldsymbol{\rho}\right) \boldsymbol{F}_{\omega, k}
$$

where the vectors $v_{p}^{2}, v_{s}^{2}$ and $\rho$ correspond to the spatial distribution of the underground medium physical characteristics. Their components are equal to the squared pressure-wave velocity, the squared shear-wave velocity and the density at each point of the discretization grid respectively.

Eq. (1) includes the impedance matrix $\mathbf{A}_{\omega}$ that depends linearly on $v_{p}^{2}, v_{s}^{2}$ and $\boldsymbol{\rho}$. To accelerate the computation of $\mathbf{A}_{\omega}$, the following relation has been introduced ${ }^{1}$ :

$$
\begin{aligned}
\mathbf{A}_{\omega}\left(\boldsymbol{v}_{p}^{2}, \boldsymbol{v}_{s}^{2}, \boldsymbol{\rho}\right) & =\mathbf{H}_{\omega}^{p} \operatorname{Diag}\left\{\boldsymbol{v}_{p}^{2} \odot \boldsymbol{\rho}\right\} \mathbf{G}_{\omega}^{p} \\
& +\sum_{n=1}^{3} \mathbf{H}_{\omega, n}^{s} \operatorname{Diag}\left\{\boldsymbol{v}_{s}^{2} \odot \boldsymbol{\rho}\right\} \mathbf{G}_{\omega, n}^{s} \\
& +\omega^{2} \operatorname{Diag}\{\mathbf{M} \boldsymbol{\rho}\}
\end{aligned}
$$

where $\mathbf{G}_{\omega}^{p}, \mathbf{H}_{\omega}^{p},\left(\mathbf{G}_{\omega, n}^{s}\right)_{n=1,2,3}$ and $\left(\mathbf{H}_{\omega, n}^{s}\right)_{n=1,2,3}$ are sparse matrices corresponding to spatial filtering operators.

\subsection{Configuration considered for the tests}

In this article, we focus on the resolution of the inverse problem. Three approaches are presented. The first one (introduced in Section 2) is based on a regularization scheme

\footnotetext{
${ }^{1} \odot$ denotes the componentwise product and Diag $\{\boldsymbol{w}\}$ is a diagonal matrix with $\boldsymbol{w}$ as main diagonal
} 


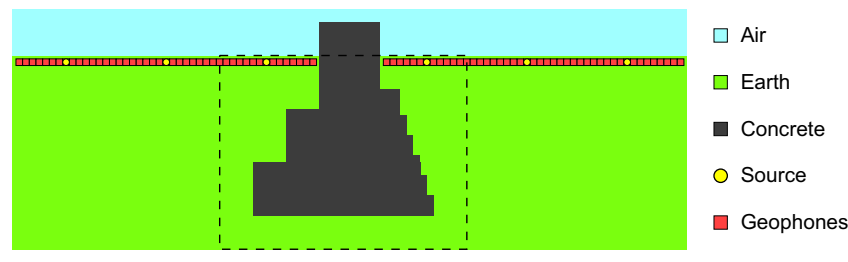

Figure 1: Scheme of the configuration considered to compare the performance of the proposed inversion methods

Table 1: Physical characteristics of the underground medium

\begin{tabular}{|c|c|}
\hline \multicolumn{1}{|c|}{ Earth } & Concrete \\
\hline$v_{E, p}=300 \mathrm{~m} / \mathrm{s}$ & $v_{C, p}=4000 \mathrm{~m} / \mathrm{s}$ \\
$v_{E, s}=150 \mathrm{~m} / \mathrm{s}$ & $v_{C, s}=2200 \mathrm{~m} / \mathrm{s}$ \\
$\rho_{T}=1500 \mathrm{~kg} / \mathrm{m}^{3}$ & $\rho_{B}=2200 \mathrm{~kg} / \mathrm{m}^{3}$ \\
\hline
\end{tabular}

commonly used in image recovery. The other two (introduced in Sections 3.1 and 3.2) take into account more precise prior information about the characteristics to be estimated.

To compare the performance of these methods, the inversion algorithms were applied on the same set of synthetic measurements. A high computation cost is required if a medium of realistic dimensions is considered. Therefore, we worked on a medium of reduced size. The configuration considered here is presented on Figure 1 and the physical characteristics of earth and concrete are listed in Table 1. More details about this configuration are given below:

- the whole medium is $2 \mathrm{~m}$ in length and $1 \mathrm{~m}$ in height;

- the medium is discretized with a spatial resolution of $2 \mathrm{~cm}$;

- the procedure is repeated for 6 successive locations of the seismic source and a set of 91 geophones is used;

- the signal emitted by the source is a $200 \mathrm{~Hz}$ Ricker wavelet;

- to solve the inverse problem, 10 frequencies located in the bandwidth of the signal were used (from 100 to $500 \mathrm{~Hz}$ );

- to avoid the inverse crime, white Gaussian noise was added to the data (the signal to noise ration equals to $30 \mathrm{~dB})$.

Instead of estimating the characteristics of the whole underground medium, we focused on the inside of a zone of interest. It is delimited by a dashed line in Figure 1. Outside the zone of interest, the underground characteristics are supposed to be perfectly known. The number of unknowns is thus reduced and the inversion procedure is accelerated.

\section{Minimization of a penalized least- square criterion}

Initially, our goal was to determine the foundation geometry. However, the propagation of a wave train through the underground medium not only depends on the foundation shape but also on the earth characteristics. Given that they cannot be determined accurately beforehand, the goal of the inverse problem is to determine the spatial distribution of the underground characteristics.
The ratio between the concrete characteristics and the earth characteristics is higher for $v_{p}$ and $v_{s}$ (greater than 13) than for $\rho$ (lower than 2) (see Table 1). Therefore, as a first approximation, the characteristic $\rho$ was supposed uniform in the whole underground medium. Its value was also supposed known beforehand. In this case, the unknown of the problem are the components of $v_{p}^{2}$ and $v_{s}^{2}$ at each point of the discretization grid. They are estimated by minimizing a penalized least-square criterion:

$$
\mathcal{J}\left(v_{p}^{2}, v_{s}^{2}\right)=\sum_{\omega, k}\left\|\boldsymbol{y}_{\omega, k}-\boldsymbol{g}_{\omega, k}\left(\boldsymbol{v}_{p}^{2}, \boldsymbol{v}_{s}^{2}\right)\right\|^{2}+\phi\left(\boldsymbol{v}_{p}^{2}, \boldsymbol{v}_{s}^{2}\right)
$$

Inverse scattering problems are known to be highly illposed. To counterbalance the ill-posedness of the problem, a penalization term $\phi$ is introduced in the criterion. It enables to take prior information into account during the inversion procedure. In our case, the underground medium is known to mainly consist of two smooth regions ("Earth" and "Concrete") separated by sharp boundaries. Therefore, we opted for a smoothed approximation of total variation so that the criterion remains differentiable:

$$
\phi\left(v_{p}^{2}, v_{s}^{2}\right)=\gamma \sum_{(i, j) \in \mathrm{C}} \sqrt{\left(v_{p_{i}}^{2}-v_{p_{j}}^{2}\right)^{2}+\left(v_{s i}^{2}-v_{s j}^{2}\right)^{2}+\delta^{2}}
$$

where $\mathrm{C}$ represents the set of neighboring pixels.

The synthetic set of data $\boldsymbol{g}_{\omega, k}$ nonlinearly depends on the unknown characteristic fields $v_{p}^{2}$ and $v_{s}^{2}$ (see Eq. 1 and 2). This makes the inverse problem difficult to solve. Moreover, the proposed algorithm must be adapted for large-scale configurations (for a realistic configuration, the dimensions of the probed domain are of several meters in length and in height and it is discretized with a spatial resolution of the order of one centimeter). Therefore, the L-BFGS-B algorithm was selected to minimize the criterion [3]. This quasiNewton method is well suited to our large-scale nonlinear optimization problem since it requires a small amount of storage to approximate the Hessian.

Our first tests on synthetic data highlighted a very slow decrease of the criterion. A great number of iterations were necessary until convergence was reached, even on configurations of small dimensions. To improve the performance of the algorithm, two changes have been made:

1. The data are introduced progressively from the low to the high frequencies. That way, spatially smooth maps are produced in a first time and sharper variations of fields $v_{p}$ and $v_{s}$ are retrieved subsequently and gradually. This solution is commonly adopted in seismic tomography [4].

2. Because of the high contrast between the concrete characteristics and the earth characteristics, criterion (3) suffers from a lack of sensitivity. This results in a slow convergence of the algorithm. We proposed in [5] to make a logarithmic variable substitution in order to counterbalance the lack of sensitivity and thus to accelerate the convergence. In the following, $\chi_{p}$ and $\chi_{s}$ denote the new variables of the problem.

The results obtained with these changes are presented on Figure 2. Two initializations of the algorithm were considered: (1) the underground medium characteristics were set to the earth values and (2) the underground medium characteristics were set to the exact solution. In each case, the 

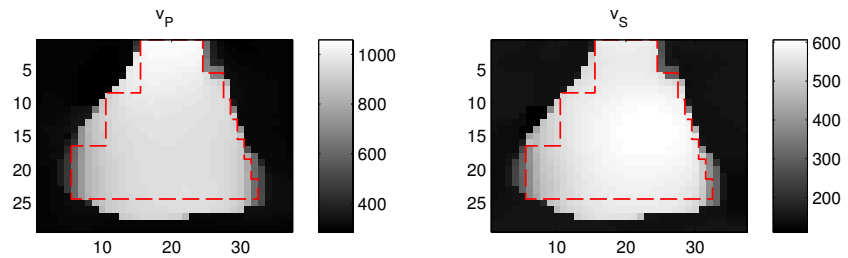

(a) Initialization to the earth characteristics
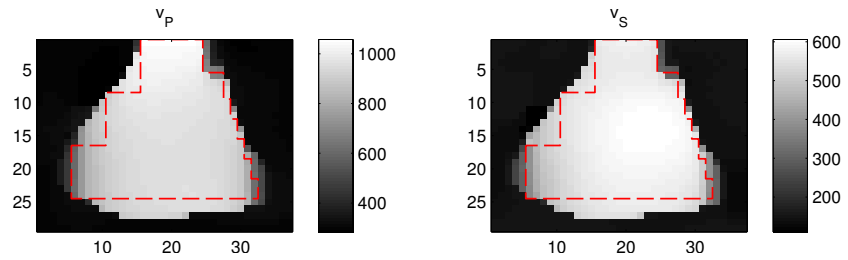

(b) Initialization to the exact solution

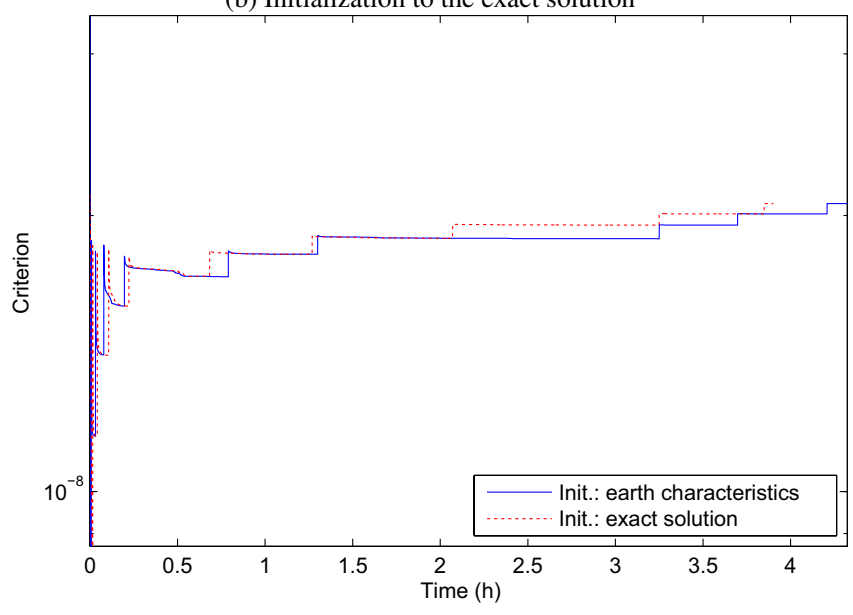

(c) Temporal evolution of the criterion

Figure 2: Results obtained using the first proposed method

estimations of the spatial distributions of $v_{p}$ and $v_{s}$ as well as the temporal evolution of the criterion is presented. The "peaks" that are visible on the criterion evolution are due to the progressive introduction of data during the inversion procedure.

For the two initializations, similar estimations of the distribution of $v_{p}$ and $v_{s}$ and quasi-identical values of the criterion were obtained after convergence. This suggests that the same minimum of the criterion was reached. Moreover, the final shape of the foundation is relatively close to the expected geometry. Nevertheless, the concrete characteristics of concrete are underestimated ( $v_{p}$ and $v_{s}$ approximately equal to $1000 \mathrm{~m} / \mathrm{s}$ and $600 \mathrm{~m} / \mathrm{s}$ instead of $4000 \mathrm{~m} / \mathrm{s}$ and 2200 $\mathrm{m} / \mathrm{s}$ respectivily) and the convergence of the algorithm remains slow.

To improve the performance of the inversion algorithm, it is necessary to incorporate more precise information about the unknowns of the problem. That is why we proposed two other approaches.

\section{Incorporation of additional prior in- formation}

\subsection{Non convex penalization}

This approach is a slight modification of the inversion method described previously. We made the assumption that the probed medium is composed of homogenous areas (earth and concrete) whose characteristics $v_{p}$ and $v_{s}$ are assumed to be estimated beforehand. A non convex penalization function $L_{2} L_{0}$ is added to the least square criterion (Eq. 3):

$$
\mathcal{J}_{L_{2} L_{0}}(\chi)=\gamma_{L_{2} L_{0}}\left(\sqrt{\delta^{2}+(\chi-\beta)^{2}}+\mu-\sqrt{\mu^{2}+(\chi-\beta)^{2}}\right)
$$

where $\delta$ and $\mu$ are scales parameters which enable to change the behaviour of the function. They have to be chosen such that: $0<\delta<\mu$. $\chi$ denote one component of $\chi_{p}$ and $\chi_{s}, \gamma_{L_{2} L_{0}}$ is a weighting parameter, and $\beta$ is the expected characteristic value which is supposed to be known beforehand.

It is assumed that adding this non-convex term to the criterion has a limited influence on the convergence of the minimization algorithm. Even if the final result is only a local minimum of the criterion, we suppose that it remains close enough to the global minimizer so that the results are still acceptable.

The $L_{2} L_{0}$ function can be seen as a "potential well". It favors the appearance of the value $\beta$ in the reconstruction result. Several $L_{2} L_{0}$ functions can be added to favor the appearance of different values of $\beta$, as shown on figure 3 . This approach has the advantage, for a slight modification of the previous inversion method, of giving similar results for a computational time significatively shorter (figure 4).

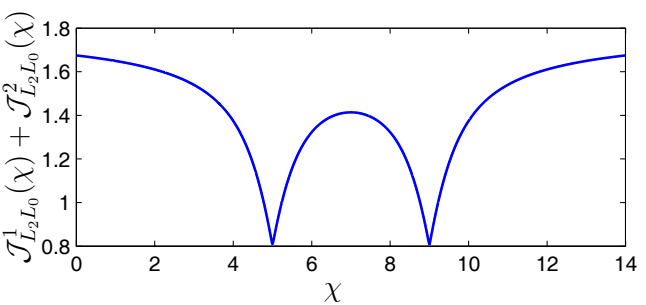

Figure 3: Sum of two $L_{2} L_{0}$ penalization functions with $\delta_{1}=\delta_{2}=0.01, \mu_{1}=\mu_{2}=0.9, \beta_{1}=5$ and $\beta_{2}=9$

\subsection{Segmentation approach}

Along with a prior estimation of the characteristics $v_{p}$ and $v_{s}$ of earth and concrete, priors about the geometry of a foundation can be taken into account during the inversion. More particularly:

- A foundation is all in one piece.

- Any horizontal cross-section corresponds to a horizontal segment in a two-dimensional representation.

- A foundation depth can be estimated beforehand with an impact-echo method [6].

To incorporate this information into the inversion algorithm, we proposed to introduce a segmentation of the probed medium into two complementary regions: a region "Earth" and a region "Concrete". For this, a new parametrization of the problem is introduced.

As for the level-set approach [7], a set of auxiliary variables is used to describe the border separating the two regions.

A finite number of horizontal lines of the discretization grid cross the foundation. Given that a prior estimation of the foundation depth is available, this number can be estimated beforehand. It is denoted by $N_{l}$ in the following. For each of 

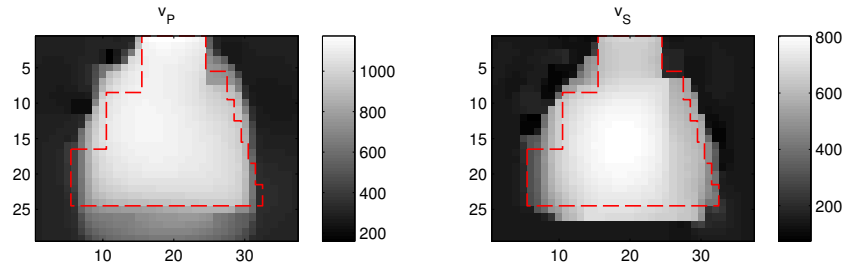

(a) Initialization to the earth characteristics
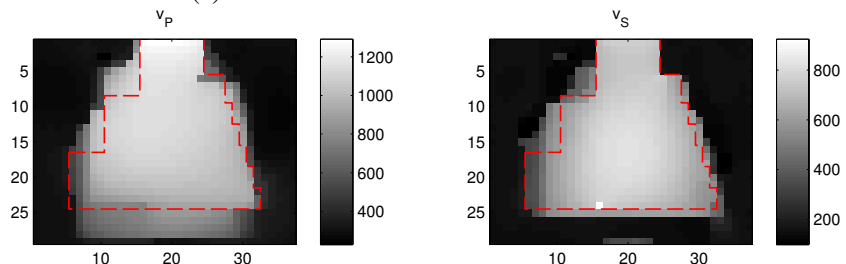

(b) Initialization to the exact solution

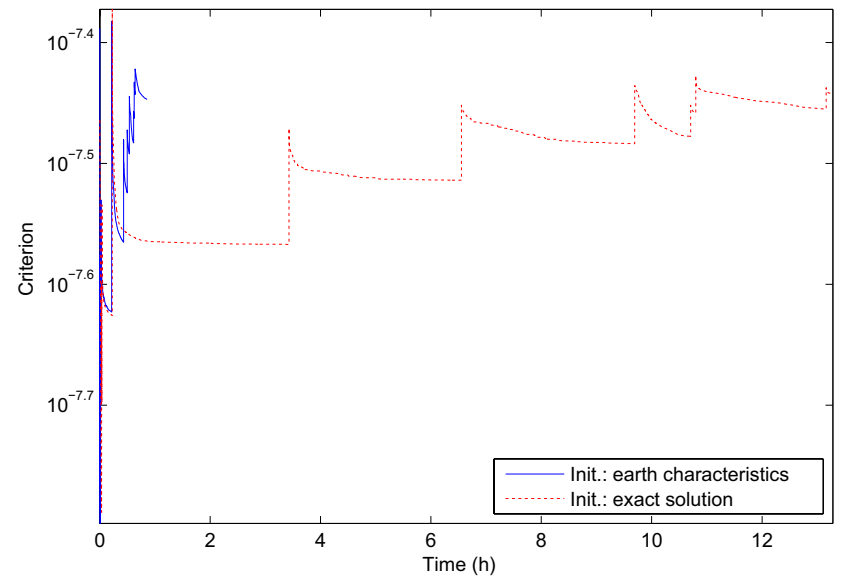

(c) Temporal evolution of the criterion

Figure 4: Results obtained with the addition of a non-convex penalization term these lines, the "Concrete" region is delimited by two transitions. Their distance form a vertical reference line crossing the foundation from top to bottom are given by two lengths $d_{l}$ and $d_{r}$ (see Figure 5). Therefore, the geometry of the foundation is totally described by two vectors $\boldsymbol{d}_{l}$ and $\boldsymbol{d}_{r}$ of length $N_{l}$.

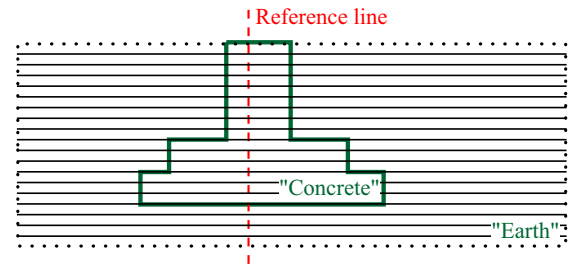

(a) Segmentation of the whole underground medium

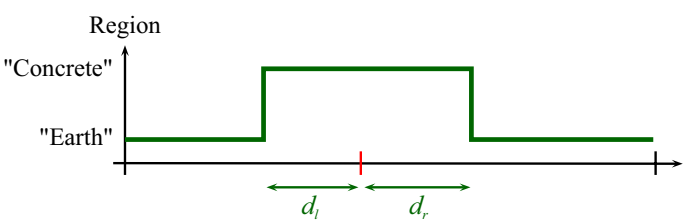

(b) Segmentation of the underground medium along each horizontal line crossing the foundation

Figure 5: Introduction of a segmentation to describe the underground medium

Two vectors $\boldsymbol{R}_{C}$ and $\boldsymbol{R}_{E}$ are introduced. They describe the segmentation of the underground medium and they depend on $\boldsymbol{d}_{l}$ and $\boldsymbol{d}_{r}$ :

$$
\begin{aligned}
\boldsymbol{R}_{C}= & 1 \text { in the region "Concrete" } \\
& 0 \text { in the region "Earth" } \\
\boldsymbol{R}_{E}= & \overline{\boldsymbol{R}_{C}}
\end{aligned}
$$

Let us denote by $\tilde{\boldsymbol{v}}_{C, p}^{e}$ and $\tilde{\boldsymbol{v}}_{C, s}^{e}$ (resp. $\tilde{\boldsymbol{v}}_{E, p}^{e}$ and $\tilde{\boldsymbol{v}}_{E, s}^{e}$ ) the prior estimations of the concrete characteristics (resp. the earth characteristics). From these prior estimations and the segmentation of the undergound medium, the characteristic filelds $\chi_{p}$ and $\chi_{s}$ are defined as follows:

$$
\begin{gathered}
\chi_{p}=\log \tilde{\boldsymbol{v}}_{E, p}^{e} \boldsymbol{R}_{E}\left(\boldsymbol{d}_{l}, \boldsymbol{d}_{r}\right)+\log \tilde{\boldsymbol{v}}_{C, p}^{e} \boldsymbol{R}_{C}\left(\boldsymbol{d}_{l}, \boldsymbol{d}_{r}\right)+\boldsymbol{\varepsilon}_{p} \\
\boldsymbol{\chi}_{s}=\log \tilde{\boldsymbol{v}}_{E, s}^{e} \boldsymbol{R}_{E}\left(\boldsymbol{d}_{l}, \boldsymbol{d}_{r}\right)+\log \tilde{\boldsymbol{v}}_{C, s}^{e} \boldsymbol{R}_{C}\left(\boldsymbol{d}_{l}, \boldsymbol{d}_{r}\right)+\boldsymbol{\varepsilon}_{s}
\end{gathered}
$$

The prior estimations may be inaccurate. To take into account the difference between the estimated and the exact underground characteristic values, the variables $\varepsilon_{p}$ and $\varepsilon_{s}$ are introduced. The underground medium characteristics are thus defined by $\boldsymbol{d}_{l}$ and $\boldsymbol{d}_{r}$ (describing the shape of the foundation) and by $\boldsymbol{\varepsilon}_{p}$ and $\boldsymbol{\varepsilon}_{s}$ (determining the characteristic values inside each region). As before, the variables are optimized by minimizing a penalized least-squares criterion where the function $\phi$ penalizes the quadratic norm of $\boldsymbol{\varepsilon}_{p}$ and $\boldsymbol{\varepsilon}_{s}$. In most cases, the width of the foundation increases with depth. This prior information was also taken into account in our tests. For that, constraints were applied on $\boldsymbol{d}_{l}$ and $\boldsymbol{d}_{r}\left([\boldsymbol{d}]_{l}^{i+1} \geq\left[\boldsymbol{d}_{l}\right]^{i}\right.$ and $\left[\boldsymbol{d}_{r}\right]^{i+1} \geq\left[\boldsymbol{d}_{r}\right]^{i}$ ).

The results are presented on Figure 6. For the first initialization of the algorithm, the top of the foundation was extended down to the estimated depth so that the constraints on $\boldsymbol{d}_{l}$ and $\boldsymbol{d}_{r}$ were satisfied from the initialization. The incorporation of strong priors into the inversion algorithm sensibly improved its performance: the final maps are very close to the expected results and the convergence was reached much faster. 

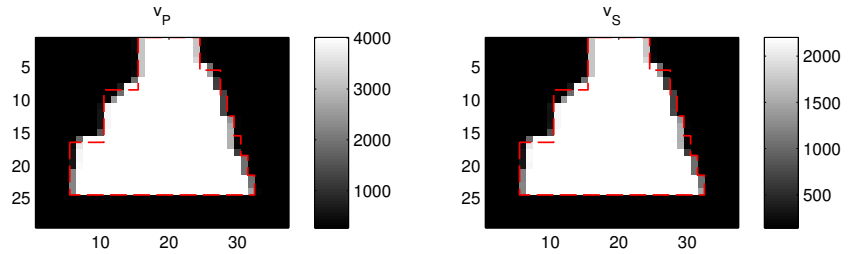

(a) Initialization: extension down to the estimated depth
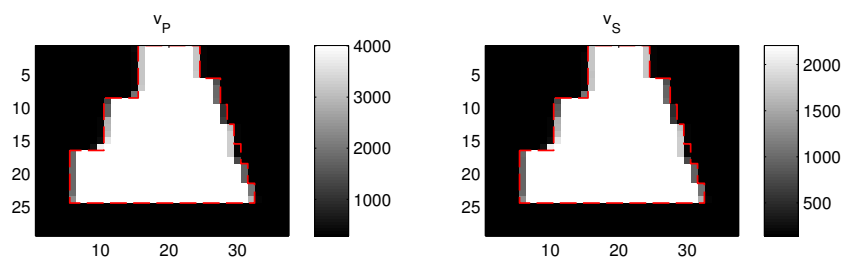

(b) Initialization to the exact solution

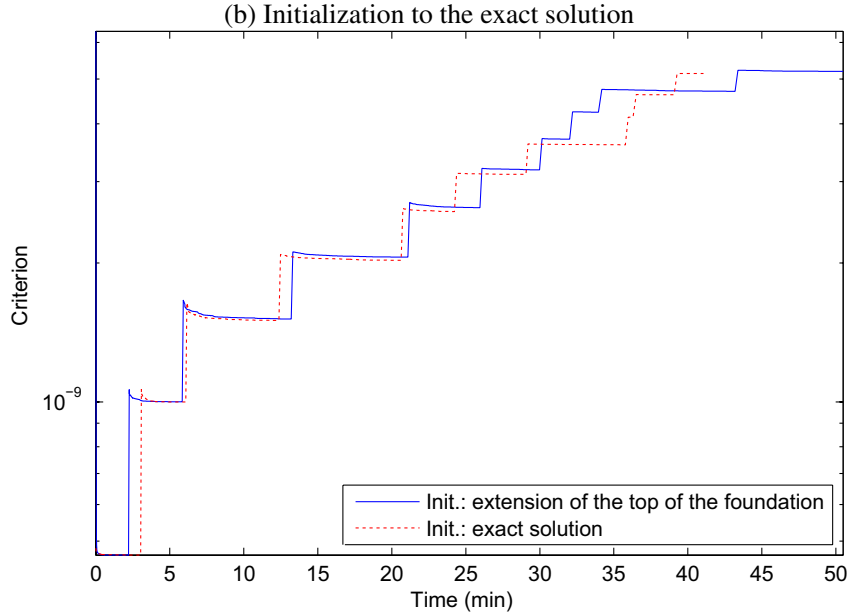

(c) Temporal evolution of the criterion

Figure 6: Results obtained with the segmentation approach

\section{Conclusion}

In this paper, three inversion procedures were presented fot the determination of the foundations geometry from scattered seismic data. The results obtained on a synthetic data set highlighted the difficulties of the problem and showed that the incorporation of precise prior information could significantly improve the performance of the inversion algorithm.

Several extensions have since been integrated to the inversion schemes. They correspond to the estimation of additional quantities:

- Tests on synthetic data showed that the hypothesis of uniformity of the density was too strong. Therefore, the estimation of its spatial distribution was included in the inversion procedure.

- To better model the attenuation of a seismic wave during its propagation in the underground medium, we worked with complex pressure- and shear-wave velocities. Their imaginary part depends on a quality factor which must be estimated during the inversion.

- The signature of the wave train propagating in the underground medium does not correspond exactly to the signal generated by the vibrator. This is due to a bad coupling between the vibrator and the ground surface. As a consequence, the source signal was also estimated.

Additional tests have been performed in order to assess the robustness of the algorithms with respect to the prior estimations (characteristics of earth and concrete, foundation depth). Tests also showed that the proposed inversion methods were efficient on configurations of realistic size. We are currently working on the treatment of real data.

\section{Acknowledgments}

The authors thank RTE - Centre National d'Expertises Réseaux for partially funding this work.

\section{References}

[1] R. Ghose, V. Nijhof, J. Brouwer, Y. Matsubara, Y. Kaida, and T. Takahashi. Shallow to very shallow, highresolution reflection seismic using a portable vibrator system. Geophysics, 63:1295-1309, 1998.

[2] L. Sirgue. The importance of low frequency and large offset in waveform inversion. In 68th EAGE Conference $\mathcal{E}$ Exhibition, 2006.

[3] C. Zhu, R. H. Byrd, and J. Nocedal. Algorithm 778: L-BFGS-B: Fortran subroutines for large-scale boundconstrained optimization. ACM Transactions on Mathematical Software, 23:550-560, 1997.

[4] R. G. Pratt. Seismic waveform inversion in the frequency domain. Part 1: theory and verification in a physical scale model. Geophysics, 64:888-901, 1999.

[5] D. Vautrin, M. Voorons, J. Idier, Y. Goussard, S. Kerzalé, and N. Paul. Seismic imaging of transmission overhead line structure foundations. In Computational Imaging IX (ISETT/SPIE Electronic Imaging), 2011.

[6] N. J. Carino. The impact-echo method: An overview. In Proc. of the Structures Congress and Exposition, 2001.

[7] S. Osher and J. A. Sethian. Fronts propagating with curvature-dependent speed: algorithms based on Hamilton-Jacobi formulations. Journal of Computational Physics, 79:12-49, 1988. 